\title{
La ley de propiedad intelectual y su repercusión en la actividad de las bibliotecas
}

\author{
Nieves Lorenzo-Escolar ${ }^{\star}$
}

\begin{abstract}
Resumen: Se analizan las repercusiones que la legislación y las nuevas tecnologías tienen en las bibliotecas en relación a la propiedad intelectual. También se examina el papel que han de tener las bibliotecas en la gestión de aspectos de la propiedad intelectual y las opciones alternativas de publicación en el ámbito académico (Open Access, repositorios)
\end{abstract}

Palabras clave: propiedad intelectual, copyright, open access, repositorios.

\section{The law of intellectual property and its impact on the activity of the libraries}

\begin{abstract}
The article analyses the impact of legislation and new technologies on libraries in relation to intellectual property. It also examines the role libraries should play in the management of intellectual property issues as well as publishing alternatives in the academic area (Open Access, repositories).
\end{abstract}

Keywords: intellectual property, copyright, open access, repositories.

\section{Introducción}

En la antigua Grecia se condenaba el plagio, entre otras cosas porque se valoraba la capacidad intelectual de cada persona y el hecho de copiar demostraba muy escaso talento.

En la época medieval cuando cada libro se copiaba a mano, proceso que duraba un largo tiempo, el derecho de autor no se valoraba. Artistas, artesanos y trabajadores no se definían como hoy en día.

En un principio, el derecho de autor, que más adelante recibirá el nombre de Propiedad Intelectual, se concibe como un derecho del editor y no del autor. Esto ocurre antes de la invención de la imprenta y también, en mayor medida, después. Este derecho no existe con carácter general, sino que se confiere mediante un privilegio que concede el monarca $-\mathrm{y}$, a veces, las universidades- a un editor o impresor determinado, mediante el cual se le permite la reproducción

* Facultad de Ciencias Económicas y Empresariales. Universidad del País Vasco UPV/EHU. Corre-e: nieves.lorenzo@ehu.es

Recibido: 11-12-08; 2. ${ }^{a}$ versión: 20-4-09. 
manuscrita o impresa de un libro con carácter exclusivo. Este privilegio, por tanto, no va dirigido a proteger la creación del libro, sino su publicación.

El autor no recibe beneficio alguno de carácter económico, pues, aunque considera lógico vivir bajo el patronazgo de un mecenas, estima que sería indigno recibir beneficios por la difusión de sus obras originales. El autor únicamente facilita la concesión del privilegio al editor y le autoriza a reproducir la obra; y estas autorizaciones — rutinarias en un principio- se convierten en elementos esenciales. Posteriormente, la competencia editorial hace que los interesados en la impresión de un determinado original ofrezcan a su autor una cantidad de dinero (o una participación en los beneficios que de la difusión se obtengan) para conseguir su autorización.

Durante los primeros 300 años de la imprenta, por tanto, la voluntad de los autores y la virtualidad de unos presuntos derechos de propiedad sobre sus obras, fue un asunto menor y marginal en la legislación.

Ésta es la situación que perdura hasta la etapa revolucionaria de finales del siglo XVIII. Los principios revolucionarios afectarán al derecho de autor: el principio de igualdad de los hombres ante la ley acabará con el sistema de los privilegios; autor y editor quedarán solos, frente a frente, a consecuencia del principio de libre contratación; la concepción de la persona como titular de derechos individuales fortalecerá al autor como titular de un derecho sobre su obra; y la exaltación del derecho de propiedad por encima de cualquier otro hará que se le considere doctrinal y legalmente como una forma más de ese derecho.

Será en el siglo XIX cuando se consolide definitivamente el reconocimiento del trabajo del autor, al tiempo que aparece el primer convenio universal de protección de los autores, firmado en Berna en 1886.

Actualmente dos sistemas sustentan el derecho de autor:

- la convención de Berna de 1886 (1) (completado en 1996 con el tratado de la OMPI sobre derecho de autor en relación a las nuevas tecnologías);

- la convención universal de Ginebra sobre derechos de autor de 1952, revisada en París en 1971 (2).

En España la regulación de la Propiedad Intelectual se iniciará con la ley de 1879 (3), que permaneció en vigor durante más de 100 años, hasta que quedó derogada en 1987 (4).

La legislación española sobre Propiedad Intelectual considera que la obra escrita pertenece al autor y que este puede o no negociar con terceros (normalmente editores) la cesión de los derechos de explotación: impresión, reproducción, distribución, adaptación y modificación.

Los autores obtienen unos beneficios por su trabajo de creación intelectual:

- unos son de carácter moral (reconocimiento de autoría e integridad de la obra), 
- otros son patrimoniales, de carácter económico (derecho de reproducción, distribución, comunicación pública, transformación, etc.).

Con todo, el derecho de autor, al igual que el derecho de propiedad, no es un derecho absoluto, y pueden limitarse por razones de interés social. Los límites de estos derechos buscan establecer un equilibrio entre los intereses de los autores, editores y fabricantes — que hacen posible la existencia de la obra con su inversión económica- y los de la sociedad, que se desarrolla a través de la investigación, y el acceso a las obras de creación intelectual.

El público no es reconocido como sujeto de derecho de las obras escritas. Este sólo goza de ciertos derechos de acceso público por medio de bibliotecas y otras instituciones, además de un pequeño número de prerrogativas de copia y distribución si sus fines son de carácter científico o privado.

En cuanto a las bibliotecas (instituciones clave para garantizar el acceso a la información y a la cultura), hay que tener claro que, aunque el libro es propiedad física de la biblioteca, no lo es, en ningún caso, la obra. Es el creador quien tiene los derechos sobre su creación. Por tanto, la biblioteca no puede ejercer ningún derecho reservado a los autores, salvo que esté recogido por ley.

Estos presupuestos se repiten invariablemente en casi todas las legislaciones europeas y también en la japonesa y en la estadounidense.

\section{Impacto de las tecnologías de la información y la comunicación. Nuevo marco legal}

En los últimos años, la propiedad intelectual se ha visto impactada por la irrupción y desarrollo de las nuevas tecnologías y redes de comunicación.

La aparición y difusión de nuevas tecnologías de reproducción y copia y los nuevos soportes han hecho necesario un replanteamiento en el marco legal que regula la protección del derecho de autor,

- para adaptar la normativa sobre propiedad intelectual a las tecnologías digitales;

- y analizar si el sistema tradicional tiene respuestas válidas para las nuevas formas de explotación de las obras y presentaciones protegidas e introducir las reformas en aquellos aspectos que resulten insuficientes.

Las dificultades del tema, que se reflejan en las diversas legislaciones de cada país, llevaron a la Unión Europea a comprender la repercusión económica que la propiedad intelectual conlleva y, en consecuencia, a asumir en su propia tarea la armonización de las legislaciones de los países miembros, a través de directivas que estos deben incorporar en sus respectivos ordenamientos.

Hasta el día de hoy, son siete las directivas europeas que se ocupan exclusivamente de aspectos de la propiedad intelectual. 
La legislación española fue incorporando estas directivas mediante leyes especiales, de tal manera que, junto con la Ley de 1987, de Propiedad Intelectual, durante algunos años convivieron con esa norma las distintas leyes de incorporación de estas directivas, hasta que el parlamento habilitó al gobierno para que refundiera en un solo texto todas las disposiciones sobre propiedad intelectual.

Así, mediante el Real Decreto Legislativo 1/1996, se aprueba el texto refundido de la Ley de Propiedad Intelectual (TRLPI) (5), que es el texto vigente en nuestros días, si bien, posteriormente, fue modificado para incorporar otra nueva directiva (2001/29/CE) (6), relativa a la armonización de los derechos de autor en la sociedad de la información, por la que — entre otras cosas - se modifican las formas de protección, legislativas y tecnológicas de las obras o creaciones a las que afectan los cambios tecnológicos (las tecnologías de la información y la comunicación facilitan la realización de actos susceptibles de vulnerar los derechos de autor).

La transposición de dicha directiva a nuestra legislación se concreta en la ley 23/2006 por la que se modifica el texto refundido de la Ley de Propiedad Intelectual (7). Son muchos los temas abordados en la ley 23/2006 que están cargados de complejidad técnica y no exentos de polémica entre los sectores afectados. En este artículo vamos a analizar los referentes a las bibliotecas

\section{Repercusiones de la ley de propiedad intelectual en las bibliotecas}

Las bibliotecas junto con otros centros de información y documentación, han constituido hasta la fecha el vehículo ideal de acceso a la cultura, derecho que supone la exigencia de prestaciones de servicios de índole cultural. Si bien a Ley de Propiedad Intelectual no constituye un obstáculo al acceso a la cultura en las bibliotecas, su alcance no reconoce las necesidades de gestión de estas instituciones y el necesario acceso al conocimiento y la información de los usuarios y son varios los aspectos preocupantes para las bibliotecas en este nuevo marco legal.

\subsection{La restricción de los límites a los derechos del autor}

La ley permite que las bibliotecas reproduzcan obras con fines de investigación. También el préstamo.

El nuevo marco legal, vía directiva de la UE, considera que es necesario restringir esos límites al derecho de autor ya que ahora las consecuencias del mal uso de esos límites (así por ejemplo, la piratería) tienen una mayor difusión.

El límite, la copia privada, siempre ha resultado polémico:

- Dificultad de encontrar un punto de equilibrio entre todos los intereses.

- Permanente evolución de la tecnología que facilita por un lado mecanismos de control de explotación y la realización de copias de mayor calidad. 
- Necesidad de diferenciar dos fenómenos: piratería/copia privada. Es necesario combatir el primero y delimitar con precisión el alcance del segundo.

En cuanto al canon por préstamo público, el tribunal de justicia de la UE considera que España no ha realizado de forma correcta la transposición de la directiva 92/100/CEE sobre alquiler y préstamo, al eximir a demasiadas instituciones del pago de remuneración a los autores por el préstamo de sus obras (8).

Los puntos que necesitan debatirse o definirse en este nuevo marco serían:

- Sistema de remuneración por el préstamo público.

- Sistema de recaudación y repartición de la remuneración que se pague.

- Sistema de remuneración a cambio de un límite al derecho de autor.

Hacen falta pues unas pautas para determinar la cuantía del canon y una regulación del límite de la copia privada, en particular, la fijación de los términos y condiciones de la compensación equitativa por la copia privada digital ya que la ley diferencia entre copia analógica y copia digital y modifica el concepto de copia privada.

\subsection{El amparo de las medidas tecnológicas de protección (ej.: sistemas anticopia) frente al ejercicio de límites}

El problema principal es que la directiva 2001/29/CE estableció una protección legal sin tener en cuenta que existían una serie de situaciones en las que la propia legislación reconocía unos límites a los derechos de los autores.

El juego de límites o excepciones que se establecen en la ley 23/2006, concretamente en sus artículos 31 a 40, es la piedra angular para el equilibrio del sistema, ya que reconoce la propiedad privada y al mismo tiempo le confiere una función social.

Las posibilidades de control que permiten las medidas tecnológicas, unidas a la protección que la propia legislación da a dichos sistemas, pueden acabar configurando una situación en la que será la voluntad del titular la que marcará cómo, cuándo y dónde podrán los usuarios utilizar sus obras.

Quedará, por tanto, en manos de la voluntad privada el uso y disfrute de las creaciones, independientemente de lo que establezca la ley.

\subsection{La necesidad de clarificar los aspectos legales de la explotación digital de las obras contenidas en sus fondos}

Éste es otro de los temas importantes para las bibliotecas. Un ejemplo significativo es lo que ha sucedido con la biblioteca digital europea. En 2005 la UE puso en marcha el proyecto i2010: bibliotecas digitales (9), (posteriormente bautizado como Europeana) con el objetivo de conseguir que el patrimonio cultural, audiovisual y científico europeo fuera accesible a todos los ciudadanos. 
A finales de 2006 se publicaron unas conclusiones sobre digitalización y accesibilidad en línea del material cultural y la conservación digital (10), en las que se reconoce que el marco legal en materia de Propiedad Intelectual es el principal obstáculo para hacer accesible la cultura europea a sus ciudadanos vía bibliotecas digitales.

El control de los nuevos documentos digitales es actualmente uno de los ámbitos que más preocupa en este ámbito profesional. La biblioteca, aunque sea poseedora de dichos documentos, no dispone de los derechos de autor de las obras.

Así pues, los aspectos legales de la explotación digital de las obras contenidas en sus fondos es otro de los temas importantes para las bibliotecas.

Las restricciones de nuestra legislación sobre derechos de autor y propiedad intelectual resultan en la práctica un freno para el desarrollo de la biblioteca digital. Si se quiere potenciar las bibliotecas digitales, va a ser necesario reformular el marco legal:

- Eliminando las barreras en la legislación nacional sobre digitalización.

- Estableciendo mecanismos para facilitar la digitalización de obras que no sean exclusivamente de uso público (caso de obras huérfanas) o obras descatalogadas.

En general, debe recalcarse el papel que juegan las licencias para determinar cuál debe ser el uso adecuado de los contenidos digitales. En España la necesidad de acuerdos con los titulares para llevar a cabo la digitalización de obras protegidas ha llevado al Centro Español de Derechos Reprográficos (CEDRO) a ofrecer la llamada "licencia digital", que tiene el inconveniente de que no es un sistema blanket licence, es decir, no hay un sistema de tarifa única.

Rebiun en su plan estratégico 2007-2010 (11) se ha planteado elaborar un documento con las características que debería cumplir una licencia para gestionar las digitalizaciones que se realizan en las bibliotecas universitarias.

Es necesario crear conciencia entre los sectores afectados y las autoridades competentes que regulan el marco legal, de la necesidad de mantener un sistema de límites que no perjudiquen la labor de nuestras instituciones, recordando el gran beneficio que genera nuestra actividad no sólo para la sociedad en su conjunto, sino también para los propios titulares de los derechos de autor.

\section{Hacia el acceso libre. Los repositorios}

Para las bibliotecas, la necesidad de:

- liberarse de la presión continua a la que son sometidas por los editores a causa de los elevados precios de las revistas,

- construir colecciones digitales sobre las que retener todos los derechos - al menos sobre los trabajadores de la institución a la que pertenecen-, 
- creación de recursos educativos abiertos (OCW, dossiers...) que faciliten la ALFIN, el aprendizaje y la enseñanza (12) y, finalmente,

- garantizar la preservación a largo plazo de los resultados de investigación de dicha institución.

Esto ha ido configurando poco a poco la necesidad de perfilar nuevas vías de difusión de las publicaciones científicas (13).

El libre acceso es entendido en este contexto como una opción alternativa para la publicación y difusión de los resultados de la comunidad académica.

$\mathrm{Al}$ igual que las publicaciones científicas, este sistema respeta los derechos de explotación de la propiedad intelectual, conserva el derecho de cita y regula la utilización del material por otros usuarios.

Este sistema ofrece numerosas ventajas respecto al sistema tradicional de publicación científica en revistas mantenidas por editoriales comerciales, y ofrece un canal para la publicación de los resultados de los investigadores más rápido, más barato, más completo y más transparente.

Rebiun, en su segunda línea estratégica, promueve la creación de contenidos y la incentivación del autoarchivo a través de los repositorios, averiguando el estado de los derechos de autor, gestionando los permisos necesarios, creando e introduciendo metadatos y guardando el trabajo en un depósito.

Un repositorio es un archivo digital de los productos intelectuales de carácter académico, accesible a los usuarios finales, tanto fuera como dentro de la institución, con pocas o ninguna barrera e interoperables.

El papel de fomento de los repositorios institucionales supone numerosas ventajas para las bibliotecas:

- La recuperación del control de los contenidos producidos por los investigadores de las instituciones.

- La reducción del gasto en la adquisición de publicaciones.

- La mejora de la imagen de la propia biblioteca en el seno de su institución como colaboradora activa en el proceso de investigación, entre otras.

Para poder depositar ficheros en un repositorio — institucional o personal-, es necesario que así se reconozca en el acuerdo que se firma con la editorial. Cuando un investigador pasa el proceso de revisión en una revista, firma un contrato en el que cede en exclusiva los derechos de explotación a la revista. Pero hay algunas excepciones en las cuales puede el autor explotar la obra, por ejemplo, el autoarchivo en webs personales o institucionales.

Estas excepciones o condiciones de cada revista son las que quedan recogidas en la lista elaborada por el grupo de trabajo Sherpa (UK) en el proyecto Romeo (14).

Se analizan los términos de copyright de las editoriales respecto al autoarchivo de los trabajos que publican. Se indica en cada caso el margen que cada 
editorial da al autor que publica en sus revistas para poder depositar su trabajo en repositorios abiertos:

- Editores amarillos: permiten almacenar en repositorios abiertos preprints.

- Editores azules: permiten almacenar en repositorios abiertos postprints.

- Editores verdes: permiten tanto pre-prints como post-prints.

- Editores blancos: no permiten almacenar en repositorios.

Por tanto, para poder depositar las publicaciones, es relevante tener en cuenta dichos términos y reconsiderar las revistas en las que se decide publicar.

ROMEO facilita la búsqueda por revista o por editorial, e incluye, en aquellas que está disponible, el texto donde se define la política de la revista sobre el copyright.

Uno de los objetivos operativos de Rebiun en el programa 2007-2010 es contactar con las editoriales y revistas científicas en España para tratar de integrar en la base de datos Sherpa / Romeo sus políticas de copyright en relación con el autoarchivo en repositorios institucionales.

Por su analogía con el proyecto Sherpa / Romeo debemos mencionar también el proyecto Dulcinea (derechos de copyright y las condiciones de auto-archivo de revistas científicas españolas) (15) cuyo objetivo es conocer las políticas editoriales de las revistas españolas respecto al acceso a sus archivos, los derechos de copyright sobre los mismos y cómo estos pueden afectar a su posterior autoarchivo en repositorios institucionales o temáticos.

Por otra parte, para que el autor pueda decidir el uso posterior de su obra ha de dotarla de las licencias que estime oportunas. Las licencias juegan un papel importante para determinar cuál debe ser el uso adecuado de los contenidos digitales. Esta bastante extendido el uso de las licencias Creative Commons (16) con las que el autor establece los criterios para la reutilización de su trabajo (reconocimiento, uso no comercial, etc.).

Actualmente Creative Commons se ha convertido en un sistema para difundir contenidos de manera abierta a través de un marco legal. Creative Commons es una organización sin ánimo de lucro que ofrece un sistema flexible de derechos de autor para el trabajo creativo.

Además, Creative Commons es una iniciativa Copyleft (propiedad intelectual alternativa) (17) [la propiedad intelectual alternativa tiene origen en los años 80 y permite una mayor difusión de las obras en canales de publicación no convencionales. Trata de impedir que las obras aportadas por autores altruistas puedan ser monopolizadas por terceros o utilizadas en forma distinta a la prevista por dichos autores].

Creative Commons da opciones a aquellos creadores que quieren que terceras personas utilicen y/o modifiquen su obra bajo determinadas condiciones. Estas condiciones son escogidas por el propio autor. Entre una obra con «todos 
los derechos reservados" o una "con ningún derecho reservado", Creative Commons propone tener "algunos derechos reservados".

Debemos mencionar también Science Commons (18), proyecto exploratorio para introducir la filosofía y actividades de Creative Commons en el dominio de la ciencia. Su propósito es estimular la creación científica a partir del uso compartido del conocimiento. Dicho uso puede ser sólo el producto de promover mecanismos legales y técnicos que contribuyan a eliminar las barreras que impiden compartir actualmente el conocimiento y los datos acumulados.

El acceso abierto se ha visto fomentado por una iniciativa relacionada, aunque distinta: la iniciativa de archivos abiertos (19) (Open Archives Iniciative, OAI, en adelante), que permite exponer los metadatos producidos por diversos proveedores de datos (revistas de acceso abierto, repositorios de contenidos, etc.) para que sean recolectados por los proveedores de servicios, que posteriormente construirán servicios de valor añadido, como análisis de citas, alertas, etc. sobre esos metadatos. El objetivo de OAI es facilitar una mayor visibilidad y difusión de esos datos en todo el mundo.

Las bibliotecas de las instituciones de investigación están muy implicadas en el proceso de desarrollo y difusión, tanto de las alternativas de acceso abierto, como de las herramientas de software empleadas para la implementación de los repositorios institucionales, incluyendo el desarrollo de esquemas de metadatos basados en OAI.

\section{Gestión del derecho de autor de las obras que nacen en las universidades}

Las universidades han de gestionar aspectos de propiedad intelectual en varias vías:

1. Estableciendo unas políticas claras respecto al acceso y preservación de la producción científica de sus investigadores, gestionando correctamente la propiedad intelectual y asesorando a sus investigadores.

2. Favoreciendo que esta producción sea lo más visible posible y recuperable, sin barreras que impidan su acceso.

3. Estableciendo los mecanismos de difusión y aprendizaje del significado de open access.

4. Familiarizando a la comunidad científica (científicos, profesores, técnicos y estudiantes) con las vías para poder disponer de su producción en abierto a través de Internet.

5. Estableciendo nuevos sistemas de evaluación científica donde se contemple el acceso y la visibilidad de la producción científica como un mérito y generando nuevos indicadores de impacto y evaluación de los recursos digitales.

6. Creando repositorios institucionales o consorciados. 
Algunas universidades están aprovechando el cambio de paradigma para recuperar el control sobre la difusión de la producción intelectual de sus investigadores y están organizando sistemas de edición de revistas al margen de las editoriales comerciales.

Para las instituciones públicas que financian la investigación, la difusión y evaluación más eficaz y eficiente de los resultados de investigación permite asentar las bases de una economía, y por ende, de una sociedad basada en el conocimiento.

\section{Papel de las bibliotecas en la gestión de derechos de autor}

Las bibliotecas y los profesionales de la documentación son parte importante de este proceso de cambio, ya que son mediadores en la recuperación de la información y acceso a las fuentes primarias de los resultados científicos, comunican documentos, crean repositorios institucionales o consorciados, elaboran estadísticas, rankings, currículums, análisis de citas, creación de redes sociales... pueden ser instrumento para que la participación de los autores sea más eficaz, creando un ambiente a favor del open access.

Las bibliotecas han de saber gestionar los aspectos sobre Propiedad Intelectual que les afecten y elaborar un manual de buenas prácticas en materia de gestión de derechos de autor. En este sentido, Rebiun ha elaborado un documento de buenas prácticas en materia de gestión de derechos de autor para bibliotecas universitarias (20). Dichas prácticas estarían referidas en el caso de los profesores a:

- Cómo citar documentos.

- Cómo proteger sus obras.

- Cómo tramitar números normalizados de publicaciones ISBN, ISSN, DOI...

- Qué deben hacer como autores para proteger al máximo sus derechos:

- Intentar, si se puede, publicar en una revista OA.

- Si no es así, publicar preferentemente sin cesión exclusiva de los derechos de copyright sobre los trabajos.

- Cómo autoarchivar sus trabajos.

- Cómo registrar su obra en el registro de la Propiedad Intelectual.

En el caso de los estudiantes, la biblioteca debe asesorar sobre

- La copia privada.

- Cómo proteger sus trabajos.

Por último, no podemos dejar de mencionar el proyecto Zwolle. Este proyecto desarrolla políticas de gestión del derecho de autor que se generan en las 
universidades orientadas a conseguir un equilibrio entre los intereses en juego. Parte de la premisa de que todos los agentes (universidades, autores, bibliotecas, usuarios y editoriales) son necesarios y de que sus diferentes intereses no tienen por qué ser contrapuestos. Cada agente necesita algunos derechos, ningún agente necesita todos los derechos.

El grupo Zwolle ha elaborado una serie de recomendaciones, tanto desde el punto de vista del autor como del editor, para llegar a un balance equilibrado sobre los derechos copyright de los artículos.

El portal Copyright Management for Scholarship recoge los trabajos de análisis de este grupo Zwolle y sus recomendaciones (21).

Las nuevas formas de regulación de la Propiedad Intelectual y la manera de afrontar su gestión por parte de universidades y bibliotecas suponen tanto un reto como una oportunidad a desarrollar en el actual proceso Bolonia, más cuando la UNESCO en su Resolución sobre Enseñanza Superior e Investigación (22), incluye el derecho a la Propiedad Intelectual dentro del marco de la libertad académica.

\section{Bibliografia}

1. Convenio de Berna para la Protección de las obras Literarias y Artísticas. De 9 de septiembre de 1886, http://www.wipo.int/treaties/es/ip/berne/trtdocs wo001.html [fecha de acceso 19-06-2009].

2. Convención Universal sobre derechos de autor. Ginebra 1952 (decreto Ley 12.088/57), http://www.bnp.gob.pe/pdf/convencion universal derechos autor.pdf [fecha de acceso 19-06-2009].

3. Ley de 10 de enero de 1879, de la propiedad intelectual, http://es.wikisource.org/wiki/ Ley de 10 de enero de 1879, de la propiedad intelectual (Espa\%C3\%B1a) [fecha de acceso 19-06-2009].

4. Ley 22/1987, de 11 de noviembre, de Propiedad Intelectual, http://www.derecho.com/1/ boe/ley-22-1987-propiedad-intelectual//fecha de acceso 19-06-2009].

5. Real Decreto Legislativo 1/1996, de 12 de abril, por el que se aprueba el Texto Refundido de la Ley de Propiedad Intelectual, http://noticias.juridicas.com/base datos/Admin/rdleg1-1996.html [fecha de acceso 19-06-2009].

6. Directiva 2001/29/CE del parlamento europeo y del Consejo de 22 de mayo de 2001 relativa a la armonización de determinados aspectos de los derechos de autor y derechos afines a los derechos de autor en la sociedad de la información, http://eur-lex. europa.eu/smartapi/cgi/sga doc?smartapi!celexapi!prod!CELEXnumdoc\&lg=es\&numdo $\mathrm{c}=32001 \mathrm{~L} 0029 \&$ model=guichett [fecha de acceso 19-06-2009].

7. Ley 23/2006, de 7 de julio, por la que se modifica el texto refundido de la Ley de Propiedad Intelectual, aprobado por el Real Decreto Legislativo 1/1996, de 12 de abril, http://sid.usal.es/idocs/F3/LYN9575/3-9575.pdf [fecha de acceso 19-06-2009].

8. Marandola, Marco: El préstamo en Europa y la Directiva 92/100/CE en El profesional de la información, 2004, 13 (6). Pags: 450-458. 
9. Comunicación de la Comisión al Parlamento Europeo, al Consejo, al Comité Económico y Social Europeo y al Comité de las Regiones - i2010: bibliotecas digitales \{SEC(2005) 1194\} \{SEC(2005) 1195\} http://eur-lex.europa.eu/smartapi/cgi/sga doc?smartapi!celexp lus!prod!DocNumber\&lg=es\&type doc $=$ COMfinal\&an doc $=2005 \& n u \quad$ doc $=465$ [fecha de acceso 19-06-2009].

10. Recomendación de la Comisión de Comunidades Europeas de 24 de agosto de 2006 sobre la digitalización y la accesibilidad en línea del material cultural y la conservación digital http://eur-lex.europa.eu/LexUriServ/LexUriServ.do?uri=OJ:L:2006:236:0028:0030 :ES:PDF [fecha de acceso 19-06-2009].

11. II Plan estratégico de Rebiun 2007-2010 http://eur-lex.europa.eu/LexUriServ/LexUriServ.do? uri=OJ:L:2006:236:0028:0030:ES:PDF [fecha de acceso 19-06-2009].

12. López Guzman, Clara: Repositorios de objetos de aprendizaje: bibliotecas para compartir y reutilizar recursos en los entornos e-learning en Biblioteca Universitaria, juliodiciembre 2006. Año/vol. 9, no 02, págs. 99-107 http://redalyc.uaemex.mx/redalyc/ pdf/285/28590202.pdf [fecha de acceso 19-06-2009].

13. Borrego, Angel: La propiedad intelectual de los resultados de la investigación en el ámbito universitario en ITEM, 2004 (38), 81-93.

14. Información sobre el grupo Sherpa y sus trabajos puede encontrarse en http://www. sherpa.ac.uk/romeo/ [fecha de acceso 19-06-2009].

15. Información sobre Dulcinea puede encontrarse en http://www.accesoabierto.net/dulcinea/ [fecha de acceso 19-06-2009].

16. Información sobre Creative Commons puede encontrarse en http://es.creativecommons.org/licencia/ [fecha de acceso 19-06-2009].

17. Información sobre el Copyleft puede encontrarse en http://www.gnu.org/copyleft/ [fecha de acceso 19-06-2009].

18. Información sobre Science Commons puede encontrarse en http://sciencecommons. org/about/ [fecha de acceso 19-06-2009].

19. Información sobre Open Archives Initiative puede encontrarse en http://www.openarchives.org/ [fecha de acceso 19-06-2009].

20. VII Workshop Rebiun sobre Proyectos Digitales, celebrado en la UNED el 18 y 19 de octubre de 2007. Buenas prácticas en materia de gestión de derechos de autor para bibliotecas universitarias, http://www.rebiun.org/doc/buenas practicas obj 2 2.doc [fecha de acceso 19-06-2009].

21. Información sobre el grupo Zwolle puede encontrarse en http://copyright.surf.nl/ copyright/ [fecha de acceso 19-06-2009].

22 Resolución sobre la Enseñanza Superior y la Investigación. Segundo Congreso Mundial de la Internacional de la Educación, Washington D.C. de 25 al 29 de julio de 1998, http://www.oit.org.pe/index.php?option=com content\&view=article\&id=1802:la-en-

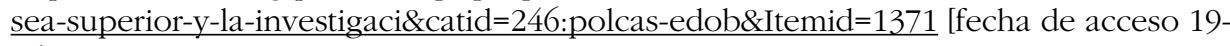
06-2009]. 